\title{
Switch da Tenofovir disoproxil fumarato (TDF) a Tenofovir alafenamide (TAF) e aumento del colesterolo: impatto sulla valutazione del rischio cardiovascolare. Switching from Tenofovir disoproxil fumarate (TDF) to Tenofovir alafenamide (TAF) and Cholesterol increase: impact on cardiovascular risk assessment.
}

Autore

per la corrispondenza:

Nicola Squillace

Infectious Diseases Unit

ASST-MONZA,

San Gerardo Hospital-

University of Milano-Bicocca,

Monza

n.squillace@asst-monza.it

Keywords:

TDF; TAF; blood lipids;

cardiovascular risk.

Potenziali conflitti

di interesse: nessuno

JHA 2019; 4(1): 12-16

DOI: 10.19198/JHA31469

Nicola Squillace ${ }^{1}$, Elena Ricci ${ }^{2}$, Barbara Menzaghi ${ }^{3}$, Guglielmo Marco Migliorino ${ }^{1}$, Giuseppe Vittorio De Socio ${ }^{4}$, Simone Passerini ${ }^{5}$, Canio Martinelli ${ }^{6}$, Maria Sabrina Mameli ${ }^{7}$, Paolo Maggi ${ }^{8}$, Katia Falasca ${ }^{9}$, Laura Cordier ${ }^{5}$, Benedetto Maurizio Celesia ${ }^{10}$, Elena Salomoni ${ }^{11}$, Antonio Di Biagio ${ }^{12}$, Giovanni Francesco Pellicano ${ }^{13}$ and Paolo Bonfanti ${ }^{14}$; per CISAI Study Group

${ }^{1}$ Infectious Diseases Unit ASST-MONZA, San Gerardo Hospital-University of Milano-Bicocca, Monza

${ }^{2}$ Fondazione IRCCS Ca' Granda Ospedale Maggiore Policlinico, Milano

${ }^{3}$ Unit of Infectious Diseases, ASST della Valle Olona - Busto Arsizio (VA)

${ }^{4}$ Department of Internal Medicine 2, Infectious Diseases Unit, "Santa Maria della Misericordia"

General Hospital, Perugia

${ }^{5}$ 1st Department of Infectious Diseases, ASST Fatebenefratelli Sacco, Milan

${ }^{6}$ SOD Malattie Infettive e Tropicali AOU Careggi, Florence

${ }^{7}$ Unit of Infectious Diseases, Department of Medical, Surgical and Experimental Sciences,

University of Sassari, Sassari

8 Infectious Disease Clinic, University of Bari, Bari.

${ }^{9}$ Clinic of Infectious Diseases, Department of Medicine and Science of Aging, University "G. d'Annunzio" Chieti-Pescara, Chieti

${ }^{10}$ Unit of Infectious Diseases University of Catania, ARNAS Garibaldi, Catania

${ }^{11}$ Infectious Diseases Unit, Santa Maria Annunziata Hospital, UsI centro, Florence

12 Infectious Diseases, San Martino Hospital Genoa, Genoa

${ }^{13}$ Department of Human Pathology of the Adult and the Developmental Age "G. Barresi",

Unit of Infectious Diseases, University of Messina, Messina, Italy

${ }^{14}$ Unit of Infectious Diseases, A. Manzoni Hospital, Lecco.

Riassunto

Lo switch da tenofovir disoproxil fumarate (TDF) a tenofovir alafenamide (TAF) è stato associato a un profondo impatto sul profile lipidico. Obiettivo di questo studio è stato valutare l'effetto della variazione dei lipidi sul rischio di malattia coronarica (CHD) e gli eventi avversi in corso di trattamento con TAF/emtricitabina/elvitegravir/cobicistat (FTC/EVG/ COBI).

Tutti i pazienti, arruolati nel progetto Surveillance Cohort

\section{Abstract}

A switch from tenofovir disoproxil fumarate (TDF) to tenofovir alafenamide (TAF) was associated with a deep impact on lipid profile. We aimed at investigating the effect of lipid variation on calculation of coronary heart disease (CHD risk) and adverse events occurring on treatment with TAF/emtricitabine/elvitegravir/cobicistat (FTC/EVG/COBI).

Consecutive HIV patients enrolled in Surveillance 
Long-term Toxicity Antiretrovirals/Antivirals (SCOLTA), che consecutivamente effettuavano lo switch da TDF/FTC/EVG/ COBI a TAF/FTC/EVG/COBI, per qualsiasi ragione, sono stati inclusi. La variazione dal baseline (TO) al follow-up a 6 mesi (T1) sono state valutate usando il t-test per dati appaiati se le differenze erano distribuite normalmente e il signed rank test se non lo erano. II rischio di CHD è stato valutato usando l'equazione di Framingham.

42 pazienti con switch da TDF/FTC/EVG/COBI a TAF/FTC/ EVG/COBI, con un' osservazione a TO per I fattori di rischio cardiovascolari sono stati inclusi in questa analisi: 23 (55\%) erano experienced e 19 (45\%) naïve agli antiretrovirali all'inizio del trattamento con TDF/FTC/EVG/COBI. Erano per il 90.5\% maschi, $93 \%$ caucasici, $88.1 \%$ in stadio CDC A o B, per il $97 \%$ con cariva virale non rilevabile. II $28 \%$ fumava al momento dell'ingresso in osservazione, mentre il $19 \%$ era composto da ex fumatori.

L'età media era 43.3 anni $( \pm 11.6)$, l'indice di massa corporea era $24.7 \pm 2,7 \mathrm{~kg} / \mathrm{m}^{2}$, il colesterolo totale (TC) $198 \pm 39.9$ $\mathrm{mg} / \mathrm{dl}$, High Density lipoprotein-cholesterol (HDL-c) $49.8 \pm$ $20.1 \mathrm{mg} / \mathrm{dl}$, la pressione arteriosa sistolica $120.5 \pm 10.3 \mathrm{mmHg}$, la mediana dei CD4 649 cell/ $\mu \mathrm{L}$ (range interquartile - IQR 507-914), la durata di ART precedente 4 anni (IQR 1.6-12.1), i trigliceridi (TRG) 110 (IQR 86-158) mg/dl, Low density lipoprotein-cholesterol (LDL-c) 123 (IQR 99-143) mg/dl. II rischio CHD era $4 \%$ (IQR 1-8\%). II tempo mediano in trattamento con TAF/ FTC/EVG/COBI è stato 199.5 (IQR 162-331). Durante il periodo di osservazione, si sono verificate 3 gg interruzioni del trattamento: una per amenorrea, una per epatite e miosite, una per un decesso causato da aplasia midollare. Un paziente è stato perso al follow-up.

At T1, TC era aumentato in maniera significativa (in media +10 $\pm 26.4 \mathrm{mg} / \mathrm{dl}, \mathrm{p}=0.02$ ) specialmente nei pazienti con switch a TAF/FTC/EVG/COBI come seconda linea di trattamento (+19 $\pm 25.8 \mathrm{mg} / \mathrm{dl}, \mathrm{p}=0.006)$. Non abbiamo osservato variazioni significative nel colesterolo HDL-c $(+2.6 \pm 15.8 \mathrm{mg} / \mathrm{dl}, \mathrm{p}=0.30)$, LDL-c ( $+4.3 \pm 33.6 \mathrm{mg} / \mathrm{dl}, \mathrm{p}=0.42)$ e nei TRG $(+16.4 \pm 84.7 \mathrm{mg} /$ $\mathrm{dl}, \mathrm{p}=0.23)$. II rischio CHD non è cambiato nel periodo di osservazione $(+0.5 \pm 2.5 \%, p=0.19)$.

Nei pazienti che hanno effettuato uno switch da TDF a TAF è stato rilevato un amento significativo del TC, senza variazioni importanti in HDL-c, LDL-c e TRG. Nonostante non si sia osservato nessun impatto sul rischio CHD nei 6 mesi successivi allo switch, per valutare l'impatto reale di questa variazione di TC sul rischio cardiovascolare nella real-life, è necessario un periodo di osservazione più lungo e un campione più numeroso.
Cohort Long-term Toxicity Antiretrovirals/Antivirals (SCOLTA) project switching from TDF/FTC/EVG/COBI to TAF/FTC/EVG/COBI for any reasons were included. Changes from baseline (TO) to 6-month follow-up (T1) were evaluated using paired t-test if differences were normally distributed and using signed rank test if not. CHD risk was evaluated by Framingham equation.

42 patients (pts) who switched from TDF/FTC/EVG/COBI to TAF/FTC/EVG/COBI with an observation at TO for cardiovascular risk factors were analyzed. 23 (55\%) pts were experienced and 19 (45\%) patients were naïve to antiretroviral drugs (ART) at the beginning of TDF/FTC/ EVG/COBI regimen. They were $90.5 \%$ male, 93\% Caucasian, $88.1 \%$ at CDC stage A-B, $97 \%$ with undetectable HIV-viral load and $28 \%$ smokers (19\% ex-smokers).

Mean age was $43.3 \pm 11.6$ years, Body Mass Index 24.7 $\pm 2,7 \mathrm{~kg} / \mathrm{m} 2$, total cholesterol (TC) $198 \pm 39.9 \mathrm{mg} / \mathrm{dl}$, High Density lipoprotein-cholesterol (HDL-c) $49.8 \pm$ $20.1 \mathrm{mg} / \mathrm{dl}$, Systolic Blood pressure $120.5 \pm 10.3 \mathrm{mmHg}$, median CD4 cell count 649 cell/ $\mu \mathrm{L}$ (interquartile range - IQR - 507-914), ART duration 4 (IQR 1.6-12.1) years, Triglycerides (TRG) 110 (IQR 86-158) mg/dl, Low density lipoprotein-cholesterol (LDL-c) 123 (IQR 99-143) mg/ dl. CHD risk was $4 \%$ (IQR 1-8\%). Median time on TAF/ FTC/EVG/COBI was 199.5 (IQR 162-331) days.

At T1, TC was significantly increased (mean variation $+10 \pm 26.4 \mathrm{mg} / \mathrm{dl}, \mathrm{p}=0.02$ ) especially in pts who switched to TAF/FTC/EVG/COBI as their second ART $(+19 \pm$ $25.8 \mathrm{mg} / \mathrm{dl}, \mathrm{p}=0.006)$. We did not observe a significant variation in $\mathrm{HDL}-\mathrm{c}(+2.6 \pm 15.8 \mathrm{mg} / \mathrm{dl}, \mathrm{p}=0.30)$, $\mathrm{LDL}-\mathrm{c}$ $(+4.3 \pm 33.6 \mathrm{mg} / \mathrm{dl}, \mathrm{p}=0.42)$ and TRG $(+16.4 \pm 84.7 \mathrm{mg} /$ $d l, p=0.23)$. CHD risk did not change in the period of observation ( $+0.5 \pm 2.5 \%, p=0.19)$. Three drug interruptions were observed: one for amenorrhea, one for hepatitis and myositis, one for death (caused by bone marrow aplasia). One patient was lost to follow-up. A significant increase in TC was observed in patients switching from TDF to TAF without an increase in HDL $-c$, LDL-C and TRG. No impact on CHD risk score was observed. A longer observation period with a larger sample is needed to evaluate the impact of TC increase on cardiovascular risk in a real life-setting.

\section{Introduzione}

I effetto ipolipemizzante di Tenofovir disoproxil L fumarate (TDF) è ben noto in letteratura (1-3) . Lo switch a tenofovir alafenamide (TAF) è associato generalmente ad aumento del colesterolo totale (CT) con stazionarietà del Rapporto CT/High Density lipoprotein-cholesterol (HDL-c) (4-7) con l'evidenza però di un incremento significativo del colesterolo LDL soprattut- to quando TAF viene associato a inibitori delle proteasi (PI) $(4,5,7)$. Alcuni studi segnalano un aumento significativo anche del rapporto $\mathrm{CT} / \mathrm{HDL}(7,8)$. Considerando che $\mathrm{CT}$ e HDL-c sono parametri utilizzati universalmente per valutare il rischio cardiovascolare (9-11)|'obiettivo del nostro studio è stato quello di valutare l'effetto dello switch da TDF a TAF sul calcolo del rischio cardiovascolare a 6 mesi dalla modifica del regime terapeutico. 


\begin{tabular}{l|c|c|c}
\hline & $\begin{array}{c}\text { Experienced } \\
\mathbf{n = 2 3 ( \% )}\end{array}$ & $\begin{array}{c}\text { Switch da prima linea } \\
\mathbf{n = 1 9 ( \% )}\end{array}$ & $\mathbf{p}$ \\
\hline M & $22(95.6)$ & $16(84.2)$ & 0.31 \\
Caucasici & $22(95.6)$ & $17(10.5)$ & 0.58 \\
Età (anni) & $49 \pm 8.8$ & $36.4 \pm 11.1$ & $<0.0001$ \\
Acquisizione HIV per via sessuale & $19(82.6)$ & $18(94.7)$ & 0.36 \\
HCV+ & $4(17.4)$ & $1(5.3)$ & 0.36 \\
Stadio CDC & & & \\
A & $10(43.5)$ & $17(89.5)$ & 0.0002 \\
B & $8(34.8)$ & $2(10.5)$ & 0.12 \\
C & $5(21.7)$ & 0 & 0.0002 \\
CD4 cells/mm3 & $624(410-857)$ & $758(541-935)$ & \\
Età (anni) & $49 \pm 8.8$ & $36.4 \pm 11.1$ & \\
Fumo & $12(52.3)$ & $10(52.6)$ & \\
Mai & $4(17.4)$ & $8(42.1)$ & 0.06 \\
Attuale & $7(30.4)$ & $1(5.3)$ & 0.30 \\
Ex & $204 \pm 45$ & $191 \pm 32$ & 0.44 \\
Colesterolo totale & $48 \pm 12$ & $52 \pm 27$ & 0.56 \\
Colesterolo HDL & $120 \pm 11$ & $122 \pm 9$ & 0.006 \\
Pressione arteriosa sistolica & $5.0(3.0-10.0)$ & $1.0(0.5-3)$ & 0.0001 \\
CHD score a T0 & $1.8(1.5-4.0)$ & $0.6(0.3-1.2)$ & \\
DAD CVD score a T0 & & & \\
\hline
\end{tabular}

Tabella 1. Caratteristiche clinico-epidemiologiche dei 42 pazienti analizzati nello studio al momento dello switch da TDF a TAF. Le variabili continue sono espresse come media \pm deviazione standard o come mediana (intervallo interquartile).

\section{Metodi}

II Progetto SCOLTA (Surveillance Cohort Long-Term Toxicity Antiretrovirals) è uno studio osservazionale multicentrico, iniziato nel 2002, e arruola persone con infezione da HIV che iniziano un nuovo farmaco, con l'intento di seguirle longitudinalmente e di identificare tossicità ed eventi avversi nell'utilizzo real-life dei farmaci (12). Lo studio SCOLTA è stato approvato dal Comitato Etico del centro coordinatore e dai Comitati Etici locali, e viene condotto in accordo con i principi etici della dichiarazione di Helsinki.

I pazienti sia naïve che experienced, che hanno più di 18 anni ed esprimono il consenso informato, possono essere arruolati. Le caratteristiche demografiche, cliniche e le variabili di laboratorio vengono registrate, includendo il CT, HDL-C, e Low density lipoproteincholesterol (LDL-C), e i trigliceridi (TRG). Tutte le informazioni sono raccolte in forma anonima in un database online. I pazienti vengono sottoposti a un follow-up semestrale, mentre eventuali eventi avversi vengono registrati quando avvengono.

I pazienti inclusi in questa analisi sono quelli arruolati nella coorte TDF/Emtricitabine/Elvitegravir/Cobicistat (TDF/FTC/EVG/COBI), con successivo switch a TDF/ Emtricitabine/Elvitegravir/Cobicistat (TAF/FTC/EVG/ $\mathrm{COBI})$, che avevano le informazioni necessarie a calco- lare il rischio di malattia coronarica (coronary heart disease, CHD). II rischio CHD è stato calcolato utilizzando il Framingham CHD score (10) e con equazione D:A:D (11): per valutare l'entità del cambiamento dovuto allo switch è stata mantenuta fissa l'età dei soggetti e l'abitudine al fumo presente al momento dello switch, mentre la pressione arteriosa sistolica e il profilo lipidico (e nel caso dell'equazione DAD anche le altre variabili implicate) sono stati aggiornati.

I pazienti sono stati descritti usando frequenza (e percentuale) per le variabili categoriche, media (con relativa deviazione standard) o mediana (con intervallo interquartile) per le variabili continue, a seconda che fossero distribuite normalmente o meno. La significatività delle variazioni di $\mathrm{CT}, \mathrm{HDL}-\mathrm{C}, \mathrm{LDL}-\mathrm{C}$, TRG e score CHD tra switch (TO) e primo follow-up (T1) sono state valutate utilizzando il t-test per dati appaiati o il signed rank test. Tutte le analisi sono state eseguite usando il software statistico SAS (versione 9.4, SAS Institute Inc., Cary, NC, USA).

\section{Risultati}

Sono stati arruolati 42 pazienti. Le caratteristiche della popolazione in studio sono elencate nella Tabella 1.

Tutti i pazienti avevano modificato la terapia da TDF/ 


\section{articolo originale}

FTC/EVG/COBI a TAF/FTC/EVG/COBI: 19 pazienti passavano a TAF/FTC/EVG/COBI da una prima linea terapeutica mentre 23 erano stati esposti anche ad altri regimi terapeutici (vedi tabella).

Quasi tutti i pazienti presentavano una carica virale di HIV non rilevabile (41 vs 1 ). II tempo mediano di esposizione a TDF/FTC/EVG/COBI era 730.5 gg (IQR range interquartile 561-823), mentre gli anni totali di terapia antiretrovirale era di 4 anni (1.6-12.1).

Al TO i pazienti presentavano le seguenti caratteristiche: età media 43.3 anni (DS deviazione standard \pm 11.6$)$, BMI 24,7 ( \pm 2.7$),$ CT $198.1 \mathrm{mg} / \mathrm{dl}$ ( \pm 39.9$), \mathrm{HDL} 49,8 \mathrm{mg} / \mathrm{dl}( \pm 20.1)$, Glicemia $90.5 \mathrm{mg} /$ dl ( \pm 13.9$)$, Creatinina $1,0 \mathrm{mg} / \mathrm{dl}( \pm 0.2)$, eGFR 88,4 $\mathrm{ml} / \mathrm{min}( \pm 17.1)$, Pressione arteriosa sistolica 120.05 $\mathrm{mmHg}( \pm 10.3)$, con mediana dei CD4=649 cell $/ \mathrm{mi}-$ crL (IQR 507-914), trigliceridi 110,5 mg/dl (86-158) ed LDL=123 mg/dl (99-143).

Il tempo mediano di esposizione a TAF/FTC/EVG/ COBI è stato di $199.5 \mathrm{gg}$ (IQR 162-331).

4 pazienti hanno interrotto il trattamento 1 per amenorrea, 1 per tossicità epatica/muscolare, 1 paziente è deceduto per aplasia midollare, 1 paziente è stato perso al follow up.

Al T1 il CT è incrementato in modo significativo (variazione media $+10.2 \pm 26.4 \mathrm{mg} / \mathrm{dl}, \mathrm{p}=0.02)$, l'HDL-c è rimasto stabile $(+2.6 \pm 15.8 \mathrm{mg} / \mathrm{dl}, \mathrm{p}=0.30)$, come anche i TRG (+16.4 $\pm 84.7 \mathrm{mg} / \mathrm{dl}, \mathrm{p}=0.23)$ e I'LDL-c $(+4.3 \pm 33.6 \mathrm{mg} / \mathrm{dl}, \mathrm{p}=0.42)$.

La stima del rischio cardiovascolare con CHD score non ha subito una variazione significativa (+0.5 \pm $2.5 \%, \mathrm{p}=0.19)$.

Anche andando a calcolare il rischio con l'equazione $D A D$, non sono stati osservati incrementi significativi $(+4.9 \pm 2.5 \%, p=0.25)$.

Andando a stratificare per pazienti che passavano a TAF/FTC/EVG/COBI da prima linea terapeutica si osservava un aumento più pronunciato del $\mathrm{CT}$ $(+19 \pm 25.8 \mathrm{mg} / \mathrm{dl}, \mathrm{p}=0.006)$ confermando la stabilità di HDL-c $(+3.9 \pm 22.2 \mathrm{mg} / \mathrm{dl}, \mathrm{p}=0.46)$, TRG $(+7.2 \pm$ $62.8 \mathrm{mg} / \mathrm{dl}, \mathrm{p}=0.63)$ e di LDL-c $(+13.7 \pm 36.8 \mathrm{mg} / \mathrm{dl}$, $p=0.13)$. Anche in questo caso la stima del rischio cardiovascolare non subiva modifiche significative $(+0.5 \pm 2.5 \%, p=0.31)$.

Per quanto riguarda il gruppo che passava a TAF/ FTC/EVG/COBI non da prima linea terapeutica (experienced), nessuno dei parametri lipidici si modificava in modo significativo: $\mathrm{CT}(+2.9 \pm 25.2 \mathrm{mg} / \mathrm{dl}, \mathrm{p}=0.59)$, $\mathrm{HDL}-\mathrm{c}(+1.5 \pm 7.9 \mathrm{mg} / \mathrm{dl}, \mathrm{p}=0.37), \mathrm{TRG}(+23.9 \pm 100$ $\mathrm{mg} / \mathrm{dl}, \mathrm{p}=0.27)$, e LDL-c $(-3.4 \pm 29.5 \mathrm{mg} / \mathrm{dl})$.

\section{Discussione}

Le modifiche del profilo lipidico osservate nella nostra coorte confermano sostanzialmente i dati di letteratura mostrando un incremento significativo del CT senza sostanziali modifiche delle altre frazioni lipidiche (4-6).

Le modifiche del CT non determinano però a breve, nel nostro studio, una variazione significativa della stima del rischio cardiovascolare.

In un precedente studio retrospettivo condotto in italia su 273 pazienti che introducevano TDF (13) era stata valutata la modifica del Framingham e del DAD score a 3 mesi dallo switch ed erano state riscontrate modifiche significative stimando il rischio con la formula DAD ma non calcolando il rischio secondo Framingham. Nel nostro studio non è stata osservata alcuna alterazione significativa del rischio sia con l'equazione Framingham che con la formula DAD.

In uno studio randomizzato-controllato prospettico condotto negli USA su 311 pazienti che sostituivano $A B C+3 T C$ con TDF+FTC in un regime contenente inibitori della proteasi invece, si osservava una riduzione significativa del rischio cardiovascolare calcolato con il metodo Framingham a 12 e a 48 settimane dalla introduzione di TDF (14).

Le modifiche del profilo lipidico dopo introduzione di TAF sembrano essere meno significative nei pazienti già lungamente esposti alla terapia antiretrovirale. Si potrebbe ipotizzare che il noto effetto ipolipemizzante di TDF (1-3) possa essere prolungato in chi è stato esposto maggiormente a TDF mentre tale effetto potrebbe più prontamente reversibile in chi ha assunto TDF per un breve periodo di tempo. Tale ipotesi viene in parte smentita dallo studio pilota di Tungsiripat $M$ et. al. (3) che rilevava un netto peggioramento del profilo lipidico già ad un mese di wash out da TDF soprattutto per i TRG. La nostra casistica però non è sottoposta ad un wash out ma alla prosecuzione del tenofovir sotto nuova formulazione e, quindi, gli effetti potrebbero essere differenti o diluiti nel tempo.

Il nostro studio ha diversi limiti: I'esiguità della casistica ed il relativo breve periodo di osservazione non permettono di generalizzare in merito. Il pregio dello studio è però quelli di porre l'attenzione su modifiche che comunque potrebbero impattare nel tempo sul rischio cardiovascolare delle persone che vivono con l'infezione da HIV. 


\section{articolo originale}

In conclusione i nostri dati offrono numerosi spunti di riflessione rispetto alle modifiche del profilo lipidico dei pazienti che sostituiscono TDF con TAF stimolando la valutazione dell'impatto di tali variazioni sul rischio cardiovascolare a medio e lungo termine su casistiche più ampie.

\section{BIBLIOGRAFIA}

1. Santos JR, Saumoy M, Curran A, Bravo I, Llibre JM, Navarro J, Estany C, et al. The lipid-lowering effect of tenofovir/emtricitabine: a randomized, crossover, double-blind, placebo-controlled trial. Clin Infect Dis 2015;61:403-408.

2. Valantin MA, Bittar R, de Truchis P, Bollens D, Slama L, Giral P, Bonnefont-Rousselot D, et al. Switching the nucleoside reverse transcriptase inhibitor backbone to tenofovir disoproxil fumarate + emtricitabine promptly improves triglycerides and low-density lipoprotein cholesterol in dyslipidaemic patients. J Antimicrob Chemother 2010;65:556-561.

3. Tungsiripat M, Kitch D, Glesby MJ, Gupta SK, Mellors JW, Moran L, Jones L, et al. A pilot study to determine the impact on dyslipidemia of adding tenofovir to stable background antiretroviral therapy: ACTG 5206. AIDS 2010;24:1781-1784.

4. Mills A, Crofoot G, Jr., McDonald C, Shalit P, Flamm JA, Gathe J, Jr., Scribner A, et al. Tenofovir Alafenamide Versus Tenofovir Disoproxil Fumarate in the First Protease Inhibitor-Based Single-Tablet Regimen for Initial HIV-1 Therapy: A Randomized Phase 2 Study. J Acquir Immune Defic Syndr 2015;69:439-445

5. Hodder S, Squires K, Kityo C, Hagins D, Avihingsanon A, Kido A, Jiang S, et al. Brief Report: Efficacy and Safety of Switching to Coformulated Elvitegravir, Cobicistat, Emtricitabine, and Tenofovir Alafenamide (E/C/F/TAF) in Virologically Suppressed Women. J Acquir Immune Defic Syndr 2018;78:209-213

6. Raffi F, Orkin C, Clarke A, Slama L, Gallant J, Daar E, Henry K, et al. Brief Report: Long-Term (96-Week) Efficacy and Safety After Switching From Tenofovir Disoproxil Fumarate to Tenofovir Alafenamide in HIV-Infected, Virologically Suppressed Adults. J Acquir Immune Defic Syndr 2017;75:226-231.

7. Eron JJ, Orkin C, Gallant J, Molina JM, Negredo E, Antinori A, Mills A, et al. A week-48 randomized phase-3 trial of darunavir/cobicistat/ emtricitabine/tenofovir alafenamide in treatment-naive HIV-1 patients. AIDS 2018;32:1431-1442.

8. Hagins D, Orkin C, Daar ES, Mills A, Brinson C, DeJesus E, Post FA, et al. Switching to coformulated rilpivirine (RPV), emtricitabine (FTC) and tenofovir alafenamide from either RPV, FTC and tenofovir disoproxil fumarate (TDF) or efavirenz, FTC and TDF: 96-week results from two randomized clinical trials. HIV Med 2018;19:724-733.

9. Goff DC, Jr., Lloyd-Jones DM, Bennett G, Coady S, D'Agostino RB, Sr., Gibbons R, Greenland P, et al. 2013 ACC/AHA guideline on the assessment of cardiovascular risk: a report of the American College of Cardiology/American Heart Association Task Force on Practice Guidelines. J Am Coll Cardiol 2014;63:2935-2959.

10. D'Agostino RB, Sr., Grundy S, Sullivan LM, Wilson P, Group CHDRP. Validation of the Framingham coronary heart disease prediction scores: results of a multiple ethnic groups investigation. JAMA 2001;286:180-187.

11. Friis-Moller N, Ryom L, Smith C, Weber R, Reiss P, Dabis F, De Wit S, et al. An updated prediction model of the global risk of cardiovascular disease in HIV-positive persons: The Data-collection on Adverse Effects of Anti-HIV Drugs (D:A:D) study. Eur J Prev Cardiol 2016;23:214-223.

12. Bonfanti P, Martinelli C, Ricci E, Carradori S, Parruti G, Armignacco O, Magnani C, et al. An Italian approach to postmarketing monitoring: preliminary results from the SCOLTA (Surveillance Cohort Long-Term Toxicity Antiretrovirals) project on the safety of lopinavir/ritonavir. I Acquir Immune Defic Syndr 2005;39:317-320

13. Gagliardini R, Fabbiani M, Colafigli M, D'Avino A, Mondi A, Borghetti A, Lamonica S, et al. Lipid-lowering effect and changes in estimated cardiovascular risk after switching to a tenofovir-containing regimen for the treatment of HIV-infected patients. J Chemother 2017;29:299-307.

14. Campo R, DeJesus E, Bredeek UF, Henry K, Khanlou H, Logue K, Brinson C, et al. SWIFT: prospective 48-week study to evaluate efficacy and safety of switching to emtricitabine/ten ofovir from lamivudine/abacavir in virologically suppressed HIV-1 infected patients on a boosted protease inhibitor containing antiretroviral regimen. Clin Infect Dis 2013;56:1637-1645. 